\title{
Detection of Deformable Objects in 3D Images Using Markov-Chain Monte Carlo and Spherical Harmonics
}

\author{
Khaled Khairy, Emmanuel Reynaud, and Ernst Stelzer
}

\begin{abstract}
We address the problem of segmenting 3D microscopic volumetric intensity images of a collection of spatially correlated objects (such as fluorescently labeled nuclei in a tissue). This problem arises in the study of tissue morphogenesis where cells and cellular components are organized in accord with biological role and fate. We formulate the image model as stochastically generated based on biological priors and physics of image formation. We express the segmentation problem in terms of Bayesian inference and use datadriven Markov Chain Monte Carlo to fit the image model to data. We perform an initial step in which the intensity volume is approximated as an expansion in 4D spherical harmonics, the coefficients of which capture the general organization of objects. Since cell nuclei are membrane-bound their shapes are subject to membrane lipid bilayer bending energy, which we use to constrain individual contours. Moreover, we parameterize the nuclear contours using spherical harmonic functions, which provide a shape description with no restriction to particular symmetries. We demonstrate the utility of our approach using synthetic and real fluorescence microscopy data.
\end{abstract}

\section{Introduction}

An important segmentation problem that arises in developmental biology and tissue morphogenesis research is when the image contains a collection of objects (such as fluorescently labeled organelles) that are spatially correlated in a biologically meaningful way, but whose position, number and geometry must be determined. Such a problem is complicated by individual variations in intensity, geometry, relative orientation and object boundary proximity (Figs. 1, 3a, 4a).

A powerful technique that can be used to attack this problem is the method of Bayesian inference coupled to Markov Chain Monte Carlo (MCMC) sampling [1-3]. An advantage of the Bayesian formulation is that it allows inclusion of image model priors in a flexible manner. Priors can be biological (e.g. existence of an ordered spatial arrangement, or expected distribution of geometrical properties among objects), biophysical (such as the intrinsic material properties of imaged objects) or geometrical (such as knowledge about the topology of the objects, symmetries etc.). Inclusion of these priors advantageously constrains the search space of MCMC and is expected to lead to faster and more accurate convergence.

In this paper we apply the data-driven Metropolis-Hastings Markov Chain Monte Carlo (DDMCMC) technique [4, 5] to the quantification of fluorescently labeled nuclei of Madin-Darby Canine Kidney (MDCK) cells forming an epithelium, that have been imaged using Single Plane Illumination Microscopy (SPIM) [6]. 
a

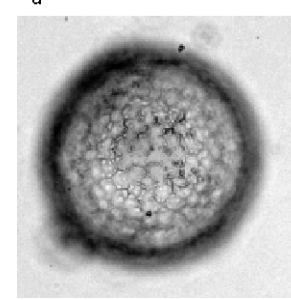

b

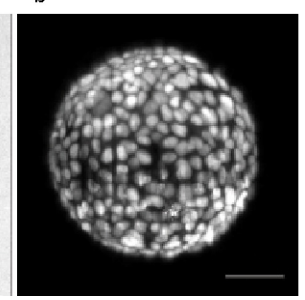

$c$

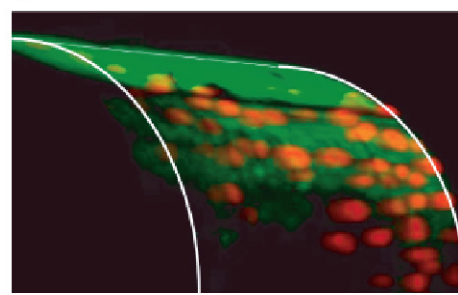

Fig. 1. Examples of spatial patterning of cells. (a) Transmitted light microscopy of a cytodex bead with an epithelium of MDCK cells, (b) Maximum projection SPIM image of fluorescently labeled (Draq5) cell nuclei of same sample as (a), bar $50 \mu \mathrm{m}$, (c) tilted maximum projection of fluorescently labeled (Draq5) nuclei of cells (red) organized within a tissue formed on a capillary tube (green -autofluorescence). White lines show the 3D geometry.

Although the methods in this paper are applicable to a wide variety of imaging modalities, 3D fluorescence microscopy, and especially SPIM, reveals highly specific spatial information (compare Fig.1a and 1b) and, when coupled to image analysis, provides insights into the $3 \mathrm{D}$ structure of cells and the organization of tissues.

\section{Theory and Computational Methods}

Briefly, the intensity volume is expanded in direction-normal spherical harmonics (4DSH), which we develop for representing 3D intensity images, to produce a "probability" image that is used to constrain the MCMC sampling process to regions where the objects are organized. We parameterize the surfaces of individual objects as a series expansion in spherical harmonic functions. The Bayesian inference problem, which incorporates bending energy constraints, is then set up, and to maximize the posterior probability we use DDMCMC. The procedure is data-driven because the MCMC sampler is explicitly constrained by the 4DSH projection.

\subsection{D Spherical Harmonics (4DSH)}

We may regard a volumetric $3 \mathrm{D}$ intensity image as a function $f$ (gray-scale intensity) of the spherical polar coordinates $\theta$ and $\phi$ in addition to the radial distance $r$ (from the image center). $f$ may be represented by $4 \mathrm{D}$ spherical harmonics as a series expansion [7],

$$
f(\theta, \phi, r)=\sum_{N=0}^{\infty} \sum_{L=0}^{\infty} \sum_{K=-L}^{L} D_{N L K}^{ \pm} H_{N L K}^{ \pm}(\theta, \phi, r)
$$

where $0<\theta<\pi$ and $0<\phi<2 \pi$, the $D_{N L K}^{ \pm}$s represent spherical harmonics coefficients indexed by integers $N, L$ and $K$ with $-L \leq K \leq L, 0 \leq L \leq \infty$ and $0 \leq N \leq \infty$, and corresponding to the basis functions $H_{N L K}^{ \pm} \mathrm{s}$, which are given by, 


$$
\begin{aligned}
& H_{N L K}^{+}(\theta, \phi, r)=A_{L K} \cdot P_{L K}(\cos \theta) \cdot \cos (K \phi) \cdot \cos \left(\frac{2 \pi N}{\tau} r\right), \text { when } \quad K \geq 0 \\
& H_{N L K}^{+}(\theta, \phi, r)=A_{L K} \cdot P_{L K}(\cos \theta) \cdot \sin (K \phi) \cdot \cos \left(\frac{2 \pi N}{\tau} r\right), \text { when } \quad K<0 \\
& H_{N L K}^{-}(\theta, \phi, r)=A_{L K} \cdot P_{L K}(\cos \theta) \cdot \cos (K \phi) \cdot \sin \left(\frac{2 \pi N}{\tau} r\right), \text { when } \quad K \geq 0 \\
& H_{N L K}^{-}(\theta, \phi, r)=A_{L K} \cdot P_{L K}(\cos \theta) \cdot \sin (K \phi) \cdot \sin \left(\frac{2 \pi N}{\tau} r\right) \quad, \text { when } \quad K<0
\end{aligned}
$$

with $A_{N L K}$ s normalization constants, $\tau$ the period in radial image space and the $P_{L K}$ representing associated Legendre polynomials.

In matrix notation, the $D_{N L K}^{ \pm} \mathrm{s}$ are determined by solving the linear system $A \cdot D=I$, where $\boldsymbol{A}$ is the matrix of basis functions $\left(H_{N L K}^{ \pm} \mathrm{s}\right), \boldsymbol{D}$ is the unknown vector of coefficients ( $D_{N L K}^{ \pm} \mathrm{s}$ ), and $\boldsymbol{I}$ is the vector of image intensities. We approximate the solution to this large dense linear least squares system using iterative residual fitting [8]. The $D_{N L K}^{ \pm}$s completely define a 3D intensity volume, and may be used to approximate the original image within series truncation limits.

\subsection{The Object Model and Spherical Harmonics Parameterization (SHP)}

The object is modeled as a closed surface filled homogenously with fluorescent dye of intensity $\lambda$. In order to avoid limiting ourselves to ellipsoids or to surfaces constrained to some particular symmetry, we chose to use the spherical harmonics parametric shape description $[9,10]$. Accordingly, a surface $\vec{S}$ that is topologically equivalent to the sphere (i.e. of genus zero), can be represented as,

$$
\vec{S}(\theta, \phi)=\left[\begin{array}{lll}
x & y & z
\end{array}\right]^{\mathrm{T}}=\left[\begin{array}{lll}
X(\theta, \phi) & Y(\theta, \phi) & Z(\theta, \phi)
\end{array}\right]^{\mathrm{T}}
$$

where $X(\theta, \phi), Y(\theta, \phi)$ and $Z(\theta, \phi)$ are coordinate functions of $\theta$ and $\phi$.

$$
X(\theta, \phi)=\sum_{L=0}^{\infty} \sum_{K=-L}^{L} C_{L K}^{X} y_{L K}(\theta, \phi)
$$

and similarly for $Y$ and $Z$. The $C_{L K}$ s are the expansion coefficients, and $y_{L K}(\theta, \phi) \mathrm{s}$ are given by

$$
\begin{aligned}
& y_{L K}(\theta, \phi)=N_{L K} P_{L, K}(\cos \theta) \cos (K \phi) \quad \text { when } \quad K \geq 0 \\
& y_{L K}(\theta, \phi)=N_{L K} P_{L, K}(\cos \theta) \sin (|K| \phi) \quad \text { when } \quad K<0
\end{aligned}
$$

where the $N_{L K}$ s are normalization constants [11]. The basis set has to be truncated at a maximum $L$ value $\left(L_{\max }\right)$ during numerical calculations. The $i^{\text {th }}$ object $s_{i}$ is 
completely parameterized by the three sets of expansion coefficients and $\lambda$ as $s_{i}=$ $\left(C_{L K}^{X}, C_{L K}^{Y}, C_{L K}^{Z}, \lambda\right)$, and a set of $n$ objects is represented by $\psi^{(n)}=\left(s_{1}, \ldots, s_{n}\right)$. Limiting the surface to ellipsoids $\left(L_{\max }=1\right)$ is still possible. To measure the correspondence between two shapes $a$ and $b$ with $N=3 \times\left(L_{\max }+1\right)^{2}$ coefficients we calculate a shape correlation coefficient $0<R_{\mathrm{a}, \mathrm{b}} \leq 1$ [9]

$$
R_{\mathrm{a}, \mathrm{b}}=1-\left(\sum_{i=1}^{N}\left(C^{\mathrm{a}, i}-C^{\mathrm{b}, i}\right)^{2}\right) /\left(\sum_{i=1}^{N}\left(\left(C^{\mathrm{a}, i}\right)^{2}+\left(C^{\mathrm{b}, i}\right)^{2}\right)\right)
$$

\subsection{The Image Model}

The image is modeled as the convolution of the unobserved image of the fluorescent dye-filled objects with the point spread function (PSF) of the microscope [2]. The PSF is approximated as a 3D Gaussian with parameters $\phi=(\alpha, \beta, \gamma)$ corresponding to the standard deviations in the xyz directions. The unobserved image is calculated by setting all voxels contained within the contours of the $n$ objects to $\lambda_{i}$, and zero otherwise. The parameter space over the collection of objects and the PSF is then given by $\Theta^{(n)}=\Psi^{(n)} \times \Phi$, and the solution space spanning all model configurations is $\Omega=\bigcup_{n>0} n \times \Theta^{(n)}$. We construct for each step in the analysis a model scene $I_{\theta}$ that corresponds to a particular configuration $\left(n, \theta^{(n)}\right) \in \Omega$.

\subsection{The Bayesian Inference Problem}

Bayesian statistical inference is used to find the most probable model $\left(n, \theta^{(n)}\right)$, i.e. that maximizes the posterior

$$
\pi\left(n, \theta^{(n)} \mid I\right) \propto L\left(I \mid n, \theta^{(n)}\right) \cdot p\left(n, \theta^{(n)}\right)
$$

where $L\left(I \mid n, \theta^{(n)}\right)$ is the likelihood of the image data $I$ given the model $\left(n, \theta^{(n)}\right)$, so assuming unit standard deviation for all voxels,

$$
L\left(I \mid n, \theta^{(n)}\right)=\prod_{i, j, k} \frac{1}{\sqrt{2 \pi}} e^{-\frac{1}{2}\left(I(i, j, k)-I_{\theta}(i, j, k)\right)^{2}}
$$

$p\left(n, \theta^{(n)}\right)$ is the model prior given by

$$
p\left(n, \theta^{(n)}\right) \propto \frac{v^{n} e^{-v}}{n !} \cdot\left(\prod_{i}^{n} f_{C_{L K}}\left(s_{i}\right) \cdot f_{E_{b}}\left(s_{i}\right) \cdot \chi\left(s_{i} \nvdash s_{j \neq i}\right)\right)
$$


where $f_{C_{L K}}\left(s_{i}\right)$ are the density functions over individual $C_{\mathrm{LK}} \mathrm{s}$, and were assumed constant over the $3\left(L_{\max }+1\right)^{2}$ shape coefficients of the $n$ objects. We assumed that the existence of an object within the image volume follows a Poisson process. This means that $n$ is Poisson distributed with intensity $v$, which is set by the user as a guess at the number of objects. The function $\chi$ is zero if overlap between two different objects is detected, 1 otherwise. The additional prior $f_{E_{b}}\left(s_{i}\right)$ is given by

$$
f_{E_{b}}\left(s_{i}\right)=\frac{1}{\sigma_{E_{\mathrm{S}}} \sqrt{2 \pi}} e^{-\frac{1}{2} \frac{\left(E_{b_{i}}-\mu_{E_{\mathrm{S}}}\right)^{2}}{\sigma_{E_{\mathrm{S}}}^{2}}} \text {, with } E_{b}=\oint_{\vec{S}}(2 H)^{2} d A
$$

where $E_{b}$ is the bending energy of the object, $A$ the surface area and $H$ the local mean curvature. $\sigma_{E_{\mathrm{S}}}$ and $\mu_{E_{\mathrm{S}}}$ are the standard deviation and mean of the expected bending energy of the objects respectively (with $\mu_{E_{\mathrm{S}}}$ set to the bending energy of the sphere) and $\gamma$ is a hyperparameter (set to 1 in this work).

\subsection{Markov Chain Monte Carlo (MCMC) Sampling}

The sampler in MCMC iteratively generates proposals within the solution space $\Omega$ by changing parameters in the previous sample. Moves are either diffusion moves; changing $C_{\mathrm{LK}} \mathrm{s}, \lambda \mathrm{s}$, or PSF parameters, or jump moves; changing the number of objects in the model, i.e. death or birth. Below we follow [2] closely.

In diffusion moves, the parameter to change is randomly selected from the existing set. The newly proposed model $\left(n, \tilde{\theta}^{(n)}\right)$ may or may not be accepted depending on how likely it is under the posterior. We replace the selected parameter's subdensity in the prior with a Gaussian that has means equal to the corresponding parameter value in the last accepted move. An acceptance probability $\alpha\left(n, \tilde{\theta}^{(n)}\right)$ is calculated for each proposal. For instance when changing an SHP coefficient of object $s_{j}$, the acceptance probability is given by,

$$
\alpha\left(n, \tilde{\theta}^{(n)}\right)=\min \left\{1, \frac{L\left(I \mid n, \tilde{\theta}^{(n)}\right)}{L\left(I \mid n, \theta^{(n)}\right)} \cdot \frac{f_{E_{b}}\left(\tilde{s}_{j}\right)}{f_{E_{b}}\left(s_{j}\right)} \prod_{i \neq j}^{n} \chi\left(s_{i} \nvdash s_{j}\right)\right\}
$$

For a jump move the dimensionality of the model is changed. In this case the acceptance probability, for instance for a birth move is calculated as

$$
\alpha\left(n, \tilde{\theta}^{(n)}\right)=\min \left\{1, \frac{L\left(I \mid n, \tilde{\theta}^{(n)}\right)}{L\left(I \mid n, \theta^{(n)}\right)} \frac{v}{n+1} \prod_{i}^{n} \chi\left(s_{i} \nvdash \tilde{s}\right)\right\}
$$

The above procedure follows the Metropolis-Hastings algorithm of MCMC [4]. 


\section{Results and Discussion}

The proposed scheme for detecting deformable objects in 3D intensity images was applied to both synthetic and real fluorescence microscopy data. For the experimental data analysis, we obtained a starting guess $\psi_{\text {starting }}^{(n)}$ by performing a small number (510) iterations of anisotropic diffusion [12] on the image, followed by the application of a marching cubes algorithm [13]. The surface triangulations obtained were individually mapped to the unit sphere and expanded in spherical harmonic functions [9, 10]. To generally describe the organization of fluorescent objects, images in all cases were expressed as a truncated 4D spherical harmonics series (Fig. 3b).

\subsection{Analysis of Synthetically Generated Data Sets}

We performed the analysis on a series of noise-free and noisy (Fig.2a) phantom im-

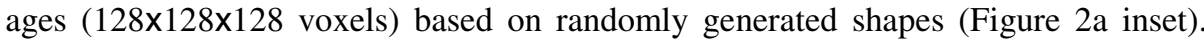
For the noisy images, the background was set to $10 \%$ of the signal intensity and photon-shot noise was simulated. Objects (1 to 15$)$ were organized in spherical fashion. 12 DDMCMC runs were performed, and terminated after 5000 iterations at a resolution of $32 \times 32 \times 32$ and 2000 iterations at the full resolution. Proposed positions (for birth moves) were based on the difference between $I_{\theta}$ and the $4 \mathrm{DSH}$ projection. The noise-free test images analysis gave geometries indistinguishable from the original (data not shown). Noisy test image results (Fig.2b) show that the degradation of recovered images stayed within acceptable ranges.

a

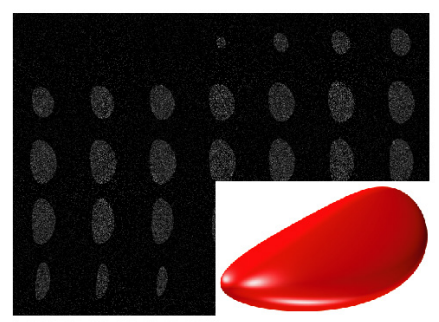

b

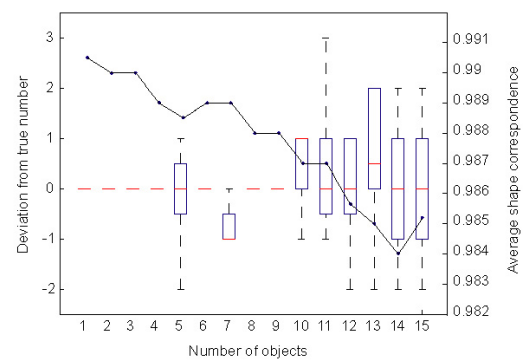

Fig. 2. Analysis of synthetic images. (a) Noisy synthetic image example and surface (inset), (b) Segmentation quality of cases 1 to 15 with noise. For boxes the lower and upper lines are the $25^{\text {th }}$ and $75^{\text {th }}$ percentiles of the sample. Whiskers indicate extent of the sample excluding outliers. The curve shows average shape correspondence (Eq.6).

\subsection{Segmentation of MDCK Cyst Nuclei}

To test the algorithm on real data, we segmented two images of MDCK cysts of different age, set 1 (Fig.3) and set 2 (Fig. 4), with $L_{\max }=2$ and the same segmentation scheme as above. Segmentation results were validated by an expert considering the number of nuclei found and the volume distribution. In set 1 the number of nuclei exactly matched the expert's decision, while in set 2 the algorithm overestimated the 
number by 4 . In both sets the volume distribution was found to be consistent with biological predictions. Moreover, the volume estimations for set 2 were a sufficient guide for the expert to identify the false positives as segmented fragments. The exact geometry of individual nuclei is more difficult to validate as there is no golden standard. Limiting $L_{\max }$ to 1 resulted in unsatisfactory segmentations for both sets, that either over- or underestimated the number of nuclei significantly (data not shown).

a

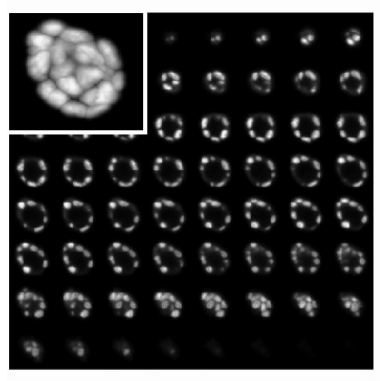

$\mathrm{b}$

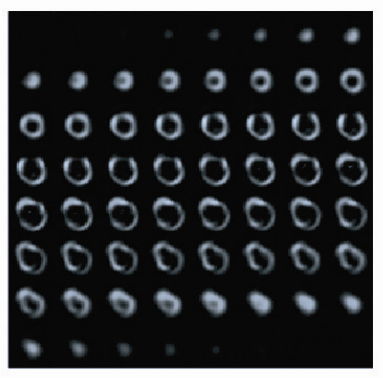

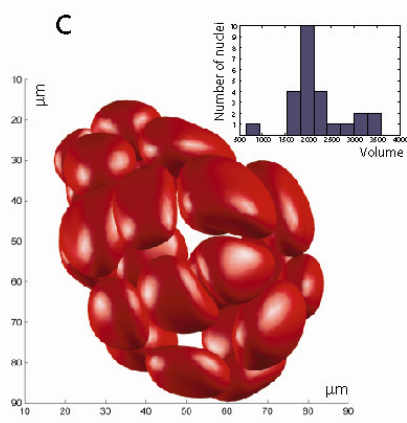

Fig. 3. 3D Analysis of MDCK cyst (one week old) image. (a) SPIM image montage frames labeled with a nuclear fluorescent marker (Draq5) (inset: projection along the z-direction). (b) 4DSH projection with $\left(N_{\max }, L_{\max }\right)=(20,10)$, (c) Segmentation result of DDMCMC, inset: nuclear volume distribution histogram.

a
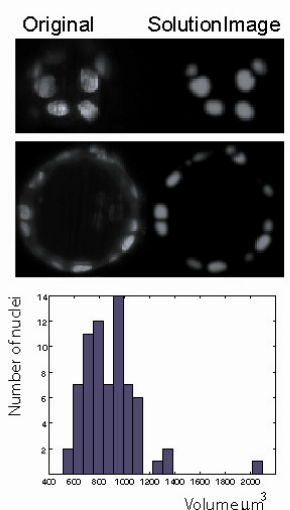

$\mathrm{b}$

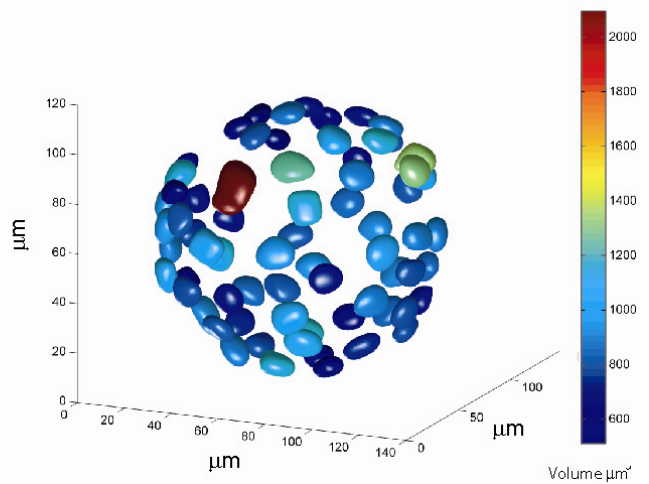

Fig. 4. Analysis of MDCK cyst (two weeks old) with fluorescently labeled nuclei (Draq5). (a) Montage pair of original data and $I_{\theta}$ frames positioned side-by-side. Bottom: nuclear volume distribution histogram, (b) Segmented surfaces. Color-coding is volume.

\section{Summary}

Segmenting fluorescently labeled structures in 3D images necessitates the incorporation of image priors to constrain the search space. In this work we formulated priors 
that are convenient and intuitive for the practitioner; namely the existence of a spatial organization, the bending energy of the labeled objects and the topology. We showed that Bayesian inference together with data-driven Markov-Chain Monte Carlo successfully fits a model image that incorporates the above information, to experimental data.

Acknowledgements. We wish to thank Philippe Girard, Petra Jakob and Philipp Keller for technical assistance. This work was funded by BMBF grant „QuantPro“ number 0313831D.

\section{References}

1. Gamerman, D., Lopes, H.F., Carlo, M.C.M.: Stochastic Simulation for Bayesian Inference. In: Carlin, B.P., et al. (eds.) Texts in Statistical Science, 2nd edn., Chapman \& Hall/CRC, Boca Raton (2006)

2. Schlecht, J., Barnard, K., Pryor, B.: Statistical Inference of Biological Structure and Point Spread Functions in 3D Microscopy. In: Third International Symposium on 3D Data Processing, Visualization and Transmission (2006)

3. Fan, A.C., et al.: MCMC Curve Sampling for Image Segmentation, in MICCAI. In: Ayache, N., Ourselin, S., Maeder, A. (eds.) MICCAI 2007, Part II. LNCS, vol. 4792, Springer, Heidelberg (2007)

4. Hastings, W.K.: Monte Carlo sampling methods using Markov Chains and their applicability. Biometrika 57, 97-109 (1970)

5. Tu, Z., Zhu, S., Shum, H.: Image segmentation by data driven Markov Chain Monte Carlo. In: International conference on computer vision, Vancouver, Canada (2001)

6. Huisken, J., et al.: Optical sectioning deep inside live embryos by selective plane illumination microscopy. Science 305(5686), 1007-1009 (2004)

7. Matheny, A., Goldgof, D.: The use of three- and four-dimensional surface harmonics for rigid and nonrigid shape recovery and representation. IEEE Transactions on Pattern Analysis and Machine Intelligence 17(10), 967-981 (1995)

8. Chung, M.K., et al.: Weighted fourier series representation and its application to quantifying the amount of gray matter. IEEE Trans. Med. Imaging 26(4), 566-581 (2007)

9. Khairy, K., Howard, J.: Spherical Harmonics-Based Parametric Deconvolution of 3D Surface Images using Bending Energy Minimization. Med. Image Anal. 12, 217-227 (2008)

10. Brechbühler, C., Gerig, G., Kuebler, O.: Parametrization of closed surfaces for 3-D shape description. Comput. Vision Image Und. 61(2), 154-170 (1995)

11. Hobson, E.W.: The theory of spherical and ellipsoidal harmonics. Chelsea, New York (1955)

12. Perona, P., Malik, J.: Scale-Space and Edge Detection Using Anisotropic Diffusion. IEEE Transactions on Pattern Recognition and Machine Intelligence 12(7), 629-639 (1990)

13. Lorensen, W.E., Cline, H.E.: A high resolution 3D surface construction algorithm. Comput. Graph. 21(4), 163-169 (1987) 